\title{
Successful Rescue Intervention of Internal Mammary Artery Anastomotic Site Acute Graft Failure With Direct New Generation Covered Stenting
}

\author{
Hassan Tahir ${ }^{\mathrm{a}, \mathrm{c}}$, James Livesay ${ }^{\mathrm{a}}$, Raj Baljepally ${ }^{\mathrm{a}, \mathrm{b}}$, Colin S. Hirst ${ }^{\mathrm{a}, \mathrm{b}}$
}

\begin{abstract}
Acute, perioperative myocardial infarction (MI) from acute left internal mammary artery (LIMA) to left anterior descending (LAD) graft failure immediately following coronary artery bypass grafting (CABG) surgery is associated with significantly increased in-hospital mortality. The leading etiology of such acute graft failure is acute thrombosis, dissection, spasm, anastomosis failure or no-reflow phenomenon. Repeat bypass surgery carries incremental risk and may not be feasible in hemodynamically unstable patients. Traditional percutaneous coronary intervention (PCI), with or without stent placement is sometimes used in such cases; however, graft anatomy and lesion location increase procedural complexity and challenge technical feasibility. This is particularly true of the LIMA to LAD graft anastomosis, where PCI carries the risk of anastomotic site perforation or avulsion. Therefore, the best revascularization strategy for such a lesion involving the LIMA to LAD graft anastomosis in the immediate perioperative period remains unknown. We present a case of 75-year-old male who suffered an acute MI complicated by cardiogenic shock less than $24 \mathrm{~h}$ after two-vessel CABG. Selective angiography revealed acute LIMA to LAD anastomotic site closure, posing a risk for perforation if treated with traditional angioplasty or stenting. We successfully performed rescue PCI, by directly deploying a PK Papyrus covered stent (Biotronik, Berlin, Germany) across the anastomosis. Our case report describes the upfront (rather than a bail out) use of the new covered stent as a novel revascularization strategy to treat "perforation prone" LIMA to LAD anastomotic site acute graft failure.
\end{abstract}

Keywords: Post-cardiotomy cardiogenic shock; Post-cardiotomy acute myocardial infarction; Acute bypass graft failure; Covered stent; PK Papyrus stent

Manuscript submitted March 10, 2021, accepted March 24, 2021

Published online May 13, 2021

aDivision of Cardiology, University of Tennessee Medical Center, Knoxville, TN, USA

${ }^{b}$ Heart Lung Vascular Institute, University of Tennessee Medical Center, Knoxville, TN, USA

${ }^{\mathrm{c} C o r r e s p o n d i n g ~ A u t h o r: ~ H a s s a n ~ T a h i r, ~ D i v i s i o n ~ o f ~ C a r d i o l o g y, ~ U n i v e r s i t y ~ o f ~}$ Tennessee Medical Center in Knoxville, Heart Lung Vascular Institute, 1940 Alcoa Hwy, Building E310, Knoxville, TN 37920, USA.

Email: doctorhassantahir@gmail.com

doi: https://doi.org/10.14740/jmc3695

\section{Introduction}

Perioperative myocardial infarction (MI) occurs in $5-10 \%$ of patients after coronary artery bypass grafting surgery $(\mathrm{CABG})$ and carries high morbidity and mortality [1]. Early graft failure remains a leading cause of acute, postoperative MI with or without the subsequent development of hemodynamic compromise or cardiogenic shock [2]. Acute graft failure management may be influenced by multiple factors like the patient's hemodynamic stability, the incremental risk of re-operation, perceived likelihood of technical or clinical success following re-operation. Management options may include emergent repeat $\mathrm{CABG}$, emergent coronary angioplasty or temporary mechanical circulatory support and possible referral to a transplant or ventricular assist device capable facility. In the setting of acute graft failure of a new bypass graft involving the left internal mammary artery (LIMA) to left anterior descending (LAD) anastomosis, the optimal revascularization strategy is unknown. The possibility of anastomotic suture site perforation or avulsion while performing traditional angioplasty/stenting would be particularly lethal, especially in an unstable patient. We present a case of acute MI complicated by cardiogenic due to acute LIMA to LAD graft closure and successful rescue percutaneous coronary intervention (PCI) by directly deploying a PK Papyrus covered stent (Biotronik, Berlin, Germany) across the distal anastomosis. We feel this strategy would not only be an appropriate rescue option but would also be "fool-proof" in preventing anastomotic site perforations, which may occur with traditional angioplasty or stenting and therefore should be routinely utilized in acute internal mammary artery (IMA) graft failure involving the anastomotic site.

\section{Case Report}

A 75-year-old male with the past medical history significant for coronary artery disease, hypertension, hyperlipidemia, insulin-dependent diabetes mellitus and end-stage renal disease on intermittent hemodialysis via a right upper extremity fistula was admitted with an acute coronary syndrome. Selective coronary angiography revealed multivessel disease, including a critical $80 \%$ stenosis of the ostium of the left main coronary artery. Transthoracic echocardiography revealed preserved left ventricular function. The patient underwent two-vessel 

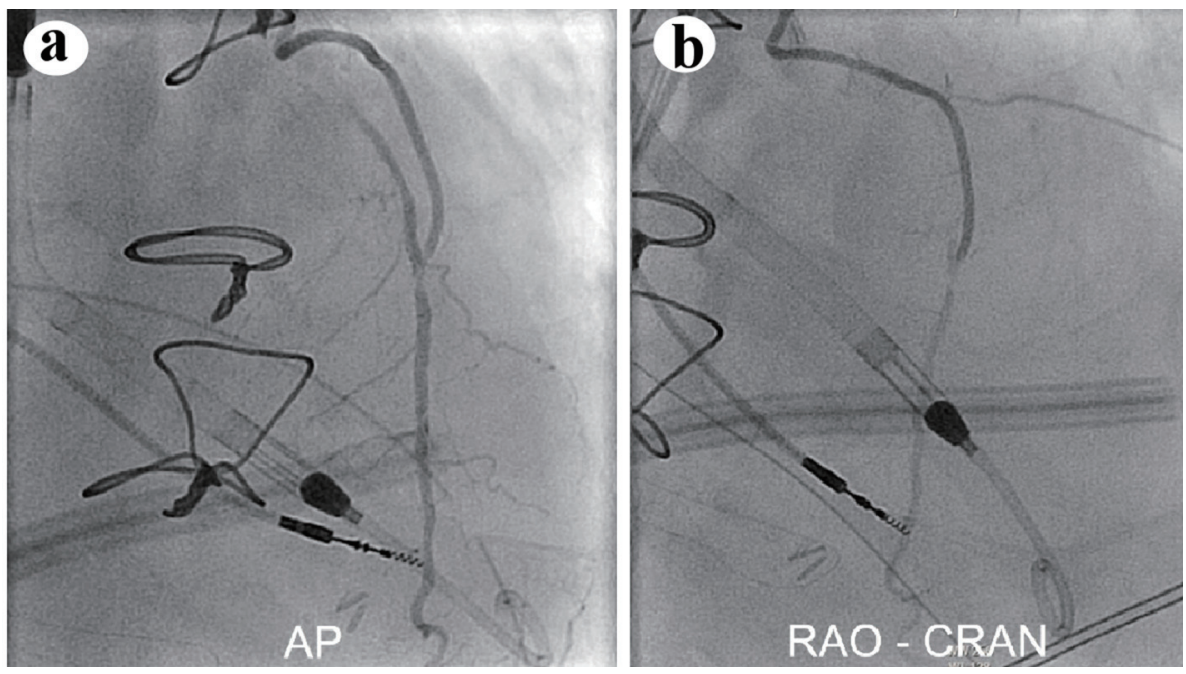

Figure 1. Selective LIMA angiography in the (a) anterior-posterior (AP) and (b) right anterior oblique/cranial (RAO-CRAN) projection showing critical, flow-limiting disease of the LIMA to LAD anastomosis and of the native LAD immediately to the graft touchdown. LIMA: left internal mammary artery; LAD: left anterior descending.

CABG: LIMA to LAD and reverse saphenous venous graft to obtuse marginal $(\mathrm{OM})$ branch with 7-0 Prolene sutures distally in end-to-side fashion to the OM and LAD, respectively.

Less than $24 \mathrm{~h}$ later, the patient developed acute respiratory distress following extubation and suffered a pulseless electrical activity cardiac arrest with successful resuscitation and return of spontaneous circulation. Following resuscitation, the patient's hemodynamic status quickly deteriorated requiring maximum vasoactive pharmacologic support. Echocardiography showed severe left ventricular systolic dysfunction and severe anterior wall hypokinesis. Patient was referred for temporary mechanical circulatory support and urgent angiography. An Impella CP device was placed and angiography revealed a $95 \%$ stenosis at the LIMA to LAD anastomosis with slow flow in the distal LAD (Fig. 1). Re-operation was not considered due to patient's poor hemodynamic status and
PCI was deemed risky given the possibility of anastomotic site perforation due to suture breakage, given that the graft was barely 1 day old. A novel strategy of direct stenting with the new covered stent platform was felt to be the most optimal technique in this setting. A 6-French IMA guide was used to engage the LIMA, a standard work-horse 0.014 -inch coronary guide wire was advanced across the lesion into the LAD, and in rapid exchange fashion, a $2.5 \times 15 \mathrm{~mm}$ PK Papyrus covered stent (Biotronik, Berlin, Germany) was advanced across the lesion (traversing the LAD to LIMA anastomosis), and successfully deployed at nominal pressure of $10 \mathrm{~atm}$. Subsequent angiography revealed antegrade Thrombolysis in Myocardial Infarction (TIMI) grade 3 flow of the LIMA and LAD with excellent myocardial blush of the subtended anterior and apical myocardial wall segments (Fig. 2). Additionally, antegrade flow of the native LAD close to the anastomosis was noted,
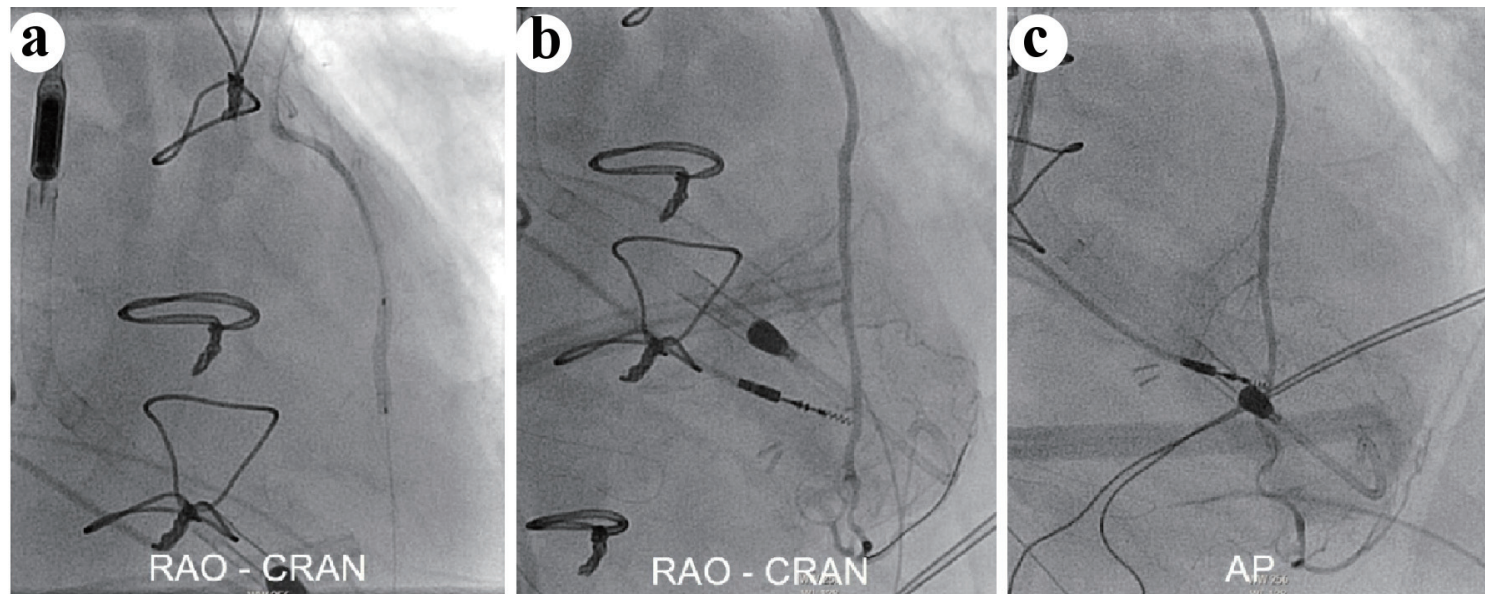

Figure 2. (a) Successful covered stent deployment across the LIMA to LAD anastomosis, followed by selective LIMA angiography in the RAO-CRAN and AP projections (b, c) showing antegrade TIMI grade 3 flow and excellent anterior and apical wall myocardial blushing. LIMA: left internal mammary artery; LAD: left anterior descending; RAO-CRAN: right anterior oblique-cranial; AP: anterior-posterior; TIMI: Thrombolysis in Myocardial Infarction. 


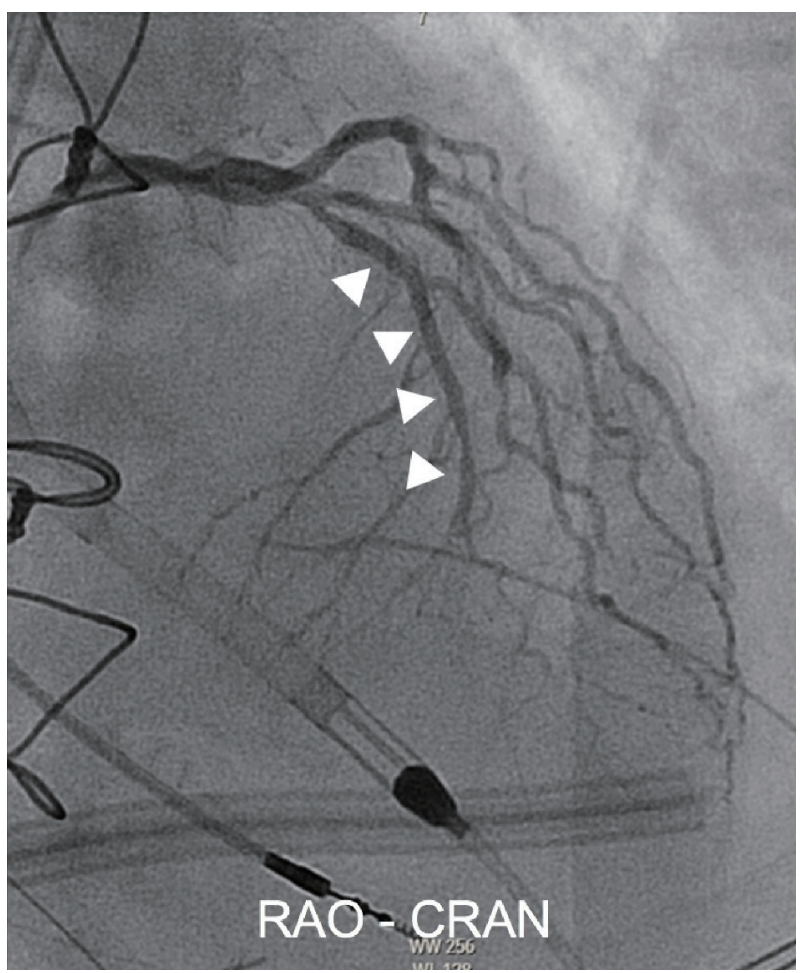

Figure 3. Selective native left coronary angiography in the RAO-CRAN projection shows excellent antegrade flow of the LAD proximal to the covered stent deployment (highlighted by arrow markings). RAOCRAN: right anterior oblique-cranial; AP: anterior-posterior; LAD: left anterior descending.

providing adequate myocardial perfusion of the basal and mid anterior wall segments (Fig. 3).

\section{Discussion}

CABG is known to improve survival in patients with left main or triple vessel disease [1]. Early graft failure post CABG, leading to perioperative MI, occurs in $5-10 \%$ of cases [2]. LIMA is the most commonly used arterial conduit for bypassing the LAD. Acute IMA graft failure is rare, and most commonly results from graft thrombosis, dissection, spasm or stenosis [3]. Data regarding the best revascularization strategy for acute IMA graft failure involving the anastomosis are lacking. Percutaneous revascularization may be preferred, especially if the patient's clinical condition may not support re-operation [4], but traditional angioplasty or stenting is fraught with risk of perforation due to loss of suture site integrity early after surgery. In this regard, several case reports have described successful PCI of a failed graft anastomosis [5, 6]. In a case series of patients who underwent urgent PCI for perioperative MI after $\mathrm{CABG}$, the rate of native coronary/bypass graft perforation due to urgent percutaneous revascularization was $30 \%$, and all patients who suffered a perforation were then subsequently treated with covered stent placement [7]. Covered stent deployment in this series was used as a bail-out treatment, a strategy that could be unforgiving in an already hemodynami- cally unstable patient, such as ours. An out-front covered stent approach will likely mitigate potential risk of anastomosis rupture, a strategy that has not been previously reported. Given the technical challenges of delivering a covered stent through a tortuous LIMA, the newer generation PK Papyrus stent, which is what we used, would be more ideal.

To our knowledge, we report the first case of a PK Papyrus direct covered stent implantation as a primary stent strategy in acute IMA graft anastomosis failure. Limitations of this strategy include lack of prior data and some reports of a higher incidence of periprocedural MI. RECOVERS trial data show an almost a three-fold higher incidence of subacute MI among patients undergoing saphenous vein grafts (SVGs) PCI with a covered stent as compared to standard bare metal stent, which may be due to subacute stent thrombosis [8]. Until recently, the Jostent Graftmaster covered stent (Abbott Cardiovascular, Abbott Park, IL) was the only commercially available covered stent approved for bail-out treatment of free perforations of native coronary arteries or SVGs $(\geq 2.75 \mathrm{~mm}$ in diameter). However, its inflexible sandwich-stent design and high crossing profile negatively impact deliverability and procedural success [9]. In comparison, the newer generation PK papyrus covered stent, membrane covered single stent design, has a slimmer crossing profile and easier maneuverability. In this regard, we believe that direct stenting with the PK Papyrus covered stent ensures technical success, and is a more appropriate technique for acute graft anastomosis failure, which can significantly reduce life-threatening complications like perforation or graft avulsion. To our knowledge no data exist regarding PK Papyrus stent longevity implanted within an arterial graft or across a graft-to-native vessel anastomosis. Although we achieved an angiographically excellent result, due to lack of experience of this stent platform in this clinical scenario, we plan to perform a selective angiography in the near future to assess anastomosis patency, irrespective of evidence of ischemia.

\section{Conclusions}

Balloon angioplasty, with or without stent deployment, at the anastomosis of a newly placed IMA graft may increase the risk of vessel perforation or anastomosis avulsion. Direct new generation covered stenting is a viable option particularly in hemodynamically unstable, inoperable patients. To our knowledge, this is the first case to describe upfront stenting of a LIMA to LAD anastomosis with a PK Papyrus covered stent. More data and collective clinical experience are needed to establish best revascularization strategy in such high risk patients.

\section{Acknowledgments}

None to declare.

\section{Financial Disclosure}

None to declare. 


\section{Conflict of Interest}

The authors report no conflict of interest.

\section{Informed Consent}

Informed consent was obtained.

\section{Author Contributions}

HT reviewed the literature and wrote the manuscript. JL provided help with the images. RB revised the manuscript and contributed to study design. $\mathrm{CH}$ revised the manuscript.

\section{Data Availability}

The authors declare that data supporting the findings of this study are available within the article.

\section{References}

1. Serruys PW, Morice MC, Kappetein AP, Colombo A, Holmes DR, Mack MJ, Stahle E, et al. Percutaneous coronary intervention versus coronary-artery bypass grafting for severe coronary artery disease. N Engl J Med. 2009; 360(10):961-972.

2. Dauerman HL, Cutlip DE, Sellke FW. Intracoronary thrombolysis in the treatment of graft closure immediate- ly after CABG. Ann Thorac Surg. 1996;62(1):280-283.

3. Nakamura T, Yamamoto S, Funayama H, Mitsuhashi T, Momomura SI. Acute occlusion of the left internal mammary artery graft in the late postoperative period. J Cardiol Cases. 2014;10(2):51-53.

4. Rasmussen C, Thiis JJ, Clemmensen P, Efsen F, Arendrup HC, Saunamaki K, Madsen JK, et al. Significance and management of early graft failure after coronary artery bypass grafting: feasibility and results of acute angiography and re-re-vascularization. Eur J Cardiothorac Surg. 1997;12(6):847-852 .

5. Hanratty CG, Koyama Y, Ward MR. Angioplasty and stenting of the distal coronary anastomosis for graft failure immediately after coronary artery bypass grafting. Am J Cardiol. 2002;90(9):1009-1011.

6. Piana RN, Adams MR, Orford JL, Popma JJ, Adams DH, Goldhaber SZ. Rescue percutaneous coronary intervention immediately following coronary artery bypass grafting. Chest. 2001;120(4):1417-1420.

7. Price MJ, Housman L, Teirstein PS. Rescue percutaneous coronary intervention early after coronary artery bypass grafting in the drug-eluting stent era. Am J Cardiol. 2006;97(6):789-791.

8. Stankovic G, Colombo A, Presbitero P, van den Branden F, Inglese L, Cernigliaro C, Niccoli L, et al. Randomized evaluation of polytetrafluoroethylene-covered stent in saphenous vein grafts: the Randomized Evaluation of polytetrafluoroethylene COVERed stent in Saphenous vein grafts (RECOVERS) Trial. Circulation. 2003;108(1):37-42.

9. GRAFTMASTER RX Coronary Stent Graft System. Index, Abbott Laboratories. www.cardiovascular.abbott/ us/en/hcp/products/percutaneous-coronary-intervention/ graftmaster-rx-stent-graft-system.html.html. 